\title{
Time-Division Multiplexing Control of Multi-Input Converters for Low-Power Solar Energy Harvesters
} \begin{abstract}
provides the highest energy yield in the worst-case scenario. Since all those panels operate at similar irradiance and temperature conditions, they can be properly biased at the same bias point by using a single maximum power point tracker (MPPT). But in those applications involving several $P V$ panels with dissimilar orientations, using an MPPT tailored to each panel would increase system cost. A better design option is to implement the MPPT with a single multiple-input converter (MIC) shared through time-division multiplexing (TDM) control. However, existing TDM controls are usually based on pulse width modulation (PWM) converters wherein the high switching frequency of transistors results in power losses that are excessive for low-power systems. Consequently, low-power MPPTs are usually based instead on pulse frequency modulation (PFM) converters. This paper proposes a novel TDM control method for MPPTs based on PFM converters.
\end{abstract} Many autonomous sensor nodes use small than secondary batteries but their energy density is much

Index Terms-Time-division multiplexing, solar energy harvesting, multiple-input converter, low-power electronics, wireless sensor networks.

\section{INTRODUCTION}

$\mathrm{S}$ olar energy harvesters are very common in autonomous sensor nodes because they rely on an inexhaustible energy source hence reduce maintenance costs with respect to primary batteries. Their lifespan, however, is limited by the degradation of energy storage devices such as secondary batteries or supercapacitors, that are needed to overcome the daily variability of sunlight. To reduce the cost of photovoltaic panels, these are conventionally oriented towards the direction that provides the highest energy yield in the worst-case scenario. Under constant daily power consumption, this scenario occurs in wintertime when average daily solar irradiation is minimal and the best tilt factor is latitude plus sun declination angle. This way, each photovoltaic (PV) panel harvests maximum daily energy and consequently, fewer panels are required. Nevertheless, some applications use PV panels with dissimilar orientations. Moveable autonomous sensor nodes, for example, such as sensor buoy systems [1], use several non-aligned PV panels to assure that the sun is shining on at least one of them. Solar energy harvesters that use supercapacitors instead of secondary batteries may also need non-aligned panels [2]. Supercapacitors have longer lifespan
than secondary batteries but their energy density is much smaller so that it is important to reduce the amount of energy to be stored in them. Aligned PV panels result in a daily power profile that rises up to a maximum and then sharply decays below the power consumed by the load $\left(P_{\text {Load }}\right)$, see Fig.1. Consequently the battery or the supercapacitor is discharging during a long time and a large amount of energy $\left(\Delta E_{\text {Bat }}\right)$ must be stored to sustain system operation. The harvested power profile can be smoothed by diverting the orientation of PV panels. Fig. 2 shows the resulting power profile and $\Delta E_{\text {Bat }}$ when two non-aligned PV panels are used. This results in a smaller daily depth of discharge (DOD) caused by $\Delta E_{\mathrm{Bat}}$ and hence the energy storage capability of the battery or the supercapacitor can be smaller. Moreover, this improves battery life because of the relationship between DOD and cycle life for Lead Acid batteries, Nickel-Metal Hydride batteries and Lithium-Metal-Polymer batteries [3]-[4].

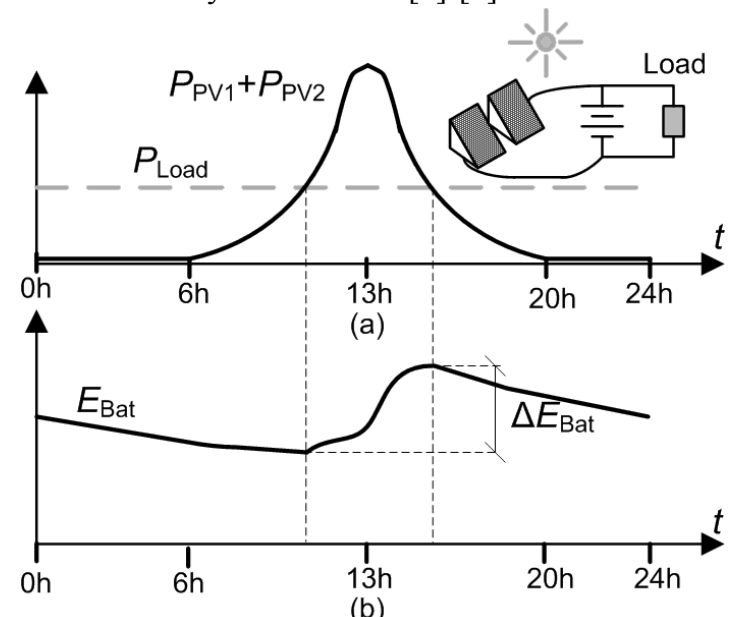

Fig. 1. (a) Daily distribution of power supplied by two PV panels $\left(P_{\mathrm{PV} 1}+P_{\mathrm{PV} 2}\right)$ sited in the North pole orientated toward South, and power consumed by the load $\left(P_{\text {Load }}\right)$; (b) energy available in the battery $\left(E_{\text {Bat }}\right)$. 


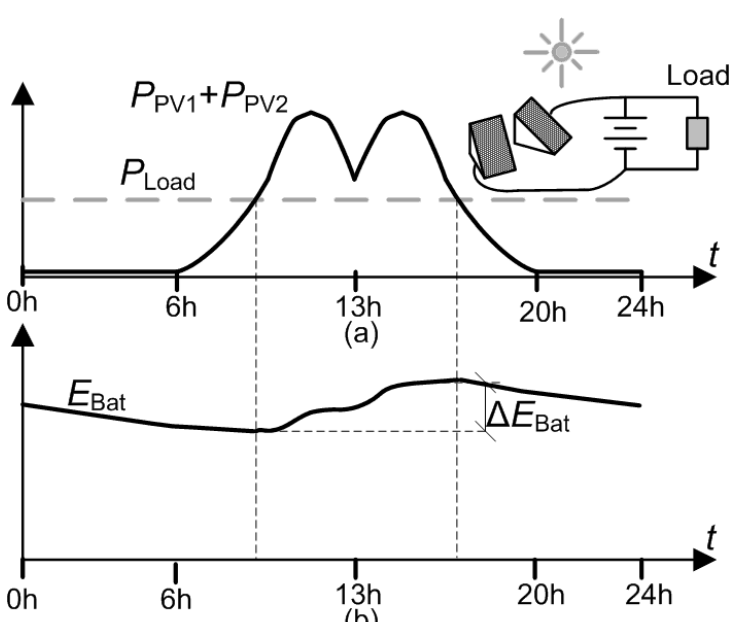

(b)

Fig. 2. (a) Daily distribution of power supplied by two PV panels $\left(P_{\mathrm{PV} 1}+P_{\mathrm{PV} 2}\right)$ sited in the North Pole, one of them orientated toward South-East and the other one toward South-West, and the power consumed by the load $\left(P_{\text {Load }}\right)$; (b) energy available in the battery $\left(E_{\mathrm{Bat}}\right)$.

Unfortunately, non-aligned PV panels increase system cost because of the increased number of PV panels and energyprocessing circuits. Since the incident irradiance on each panel is different, the resulting maximum power points (MPPs) do not match each other and an independent maximum power point tracker (MPPT) is needed for each panel. This becomes a relevant design constraint in large scale sensor networks as the final price is scaled to a large number of nodes. However, applications such as environmental monitoring, precision agriculture and smart cities do not require a high sampling rate. Typically, data is transmitted from one node to the closest one by low-power transceivers, and nodes dynamically enter or leave sleep modes according to low-power design strategies [5]. As a result, these nodes do not need high-power energy harvesters and lesser power consumption reduces costs.

This paper proposes the design of low-power low-cost solar energy harvesters for non-aligned PV panels that share a single MPPT trough a novel time-division multiplexing controller (TDM). The challenges posed by the design of this controller in low-power applications wherein Pulse Frequency Modulation (PFM) is required are analyzed in Section II.A. PFM and its advantages in low-power applications are explained in section II.b. The proposed controller and the major design constraint are exposed in Section III and IV respectively. As a proof of concept, a prototype has been implemented for two PV panels that is described in section V. Finally, conclusions are given in Section VI.

\section{BACKGROUND}

\section{A. Time-Division multiplexing of multiple-input converters}

TDM control is a common design technique in electronics that allows several systems to share a common device hence reducing cost. Multiple-input converters (MIC) are an example of TDM in power electronics wherein a single switching converter is shared by several power sources. MICs have been used in high-power applications for example to balance the state of charge (SOC) in battery-charging systems [6], to alleviate the mismatching and partial shading conditions effects in large-scale photovoltaic (PV) systems [7], to connect several renewable energy sources to a shared storage device for microgrid applications [8], and to combine two or more onboard generation units in hybrid vehicles [9].

A MIC photovoltaic system comprises several PV panels, each of them connected to a capacitor $\left(C_{1}, C_{2}, C_{3}, \ldots, C_{z_{P V}}\right)$, a multiplexer and a switching converter, see Fig. 3. The TDM control signal selects which PV panel is connected to the switching converter at a given time to transfer the energy accumulated in its associated capacitor towards the battery. Each capacitor accumulates the energy of the corresponding panel when this is not connected to the converter. The charge and discharge cycles must be controlled to held the PV panel bias voltage $\left(v_{P V 1}, v_{P V 2}, . ., v_{P V Z_{P V}}\right)$ close to the maximum power point (MPP) of the respective PV panel.

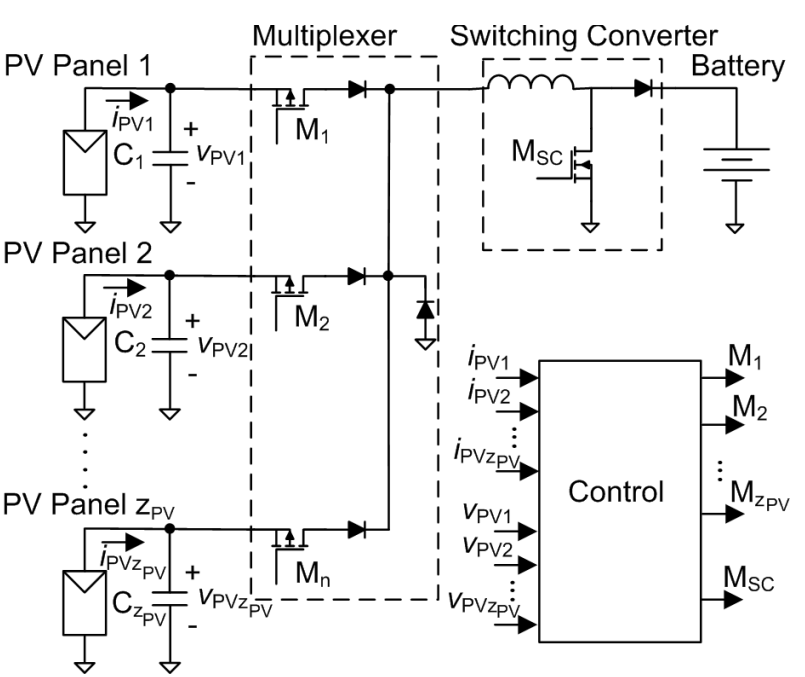

Fig. 3. Block diagram of a MIC photovoltaic system.

TDM control strategies for pulse width modulation (PWM) switching converters abound. A simple solution is the sequential connection of each PV panel during a fixed time period, which may be different for each panel [10]. During each period, the duty cycle of the transistor in the switching converter is properly tuned to regulate $\left(v_{P V 1}, v_{P V 2}, . ., v_{P V Z_{P V}}\right)$. This approach allows us to regulate the average voltage values but large fluctuation of these voltages around MPP can result hence lessening the harvested energy. Since each PV panel must be held in the charging state at least during the connection time of the other PV panels, the charging time cannot be shorter than this time interval.

To reduce the charge cycle length, the power sources can all be sequentially connected during a single switching period of the converter $(T)$ [11]-[14]. Then, during that fixed $T$ the duty cycle of each transistor is tuned according to the incoming power from PV panels. This, however, means that power switching losses are not reduced for low PV power, which is unacceptable in low-power solar energy harvesters. In those cases, $T$ must be tuned separately for each PV panel according to the generated power following a PFM control scheme. 


\section{B. PFM for low-power converters}

Switching power losses in PFM converters are reduced by shortening their switching activity [15]-[18]. The converter is hold off (Enable is OFF) while the energy from the power source is stored in a capacitor and is turned on (Enable is ON) only when the bias voltage of the capacitor $\left(v_{\mathrm{PV}}\right)$ reaches a given threshold, see Fig. 4. The converter discharges the capacitor at a constant current $\left(I_{\mathrm{DSCH}}\right)$ to keep the power source voltage within a hysteresis cycle which width is $V_{\mathrm{h}}$. Contrarily to PWM converters, PFM converters tune $T$ to keep the hysteresis window $V_{\mathrm{h}}$ fixed to a reference value around MPP $\left(V_{\mathrm{MPP}}\right)$, so that $T$ is long enough to keep a constant ratio between the switching losses and the generated PV power. Further, the switching frequency of the transistors inside the converter is high during the activation times to reduce the size of the reactive elements of the switching converter.

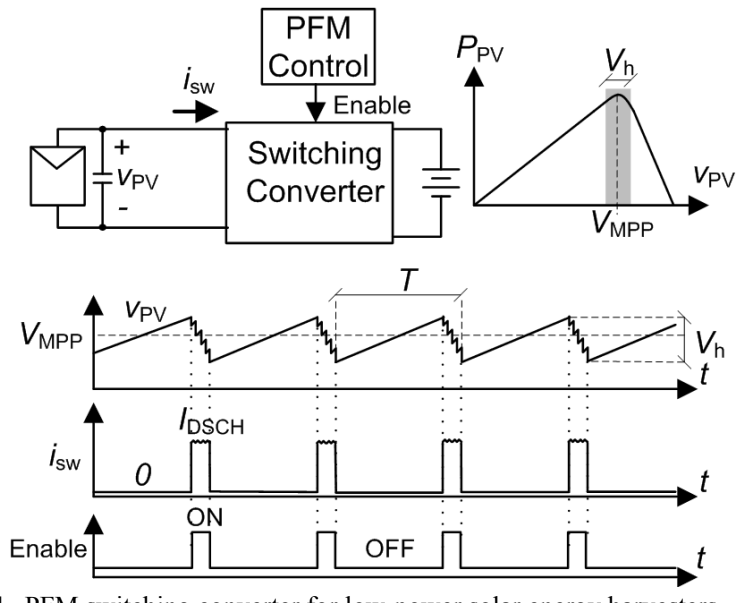

Fig. 4. PFM switching converter for low-power solar energy harvesters.

\section{PROPOSED TDM CONTROL}

The proposed control scheme distributes the discharge states of input capacitors connected to each PV panel in time to avoid overlapping. The voltage $V_{\mathrm{PV}}$ across each $\mathrm{PV}$ panel must be held at the MPP with a fixed hysteresis window. Holding a proper window $V_{\mathrm{h} \text { ref }}$ is especially important for low-power MPPT because, on the one hand, too small hysteresis windows
imply high switching activity of the converter hence high power losses, whereas, on the other hand, a too large hysteresis windows means that $V_{\mathrm{PV}}$ could be too far from MPP hence decreasing the average harvested power.

For a better comprehension, the method is firstly introduced for two PV panels and then extended to any arbitrary number of PV panels $\left(z_{\mathrm{PV}}\right)$.

\section{TDM control for two non-aligned PV panels}

Let us consider a MIC photovoltaic system such as that in Fig. 3 with two PV panels. The first step of the TDM control algorithm is to sort PV panels according to the magnitude of $i_{\mathrm{PV}}$. PV panel 1 will be that with lower $i_{\mathrm{PV}}$. The second step is to determine how many charge-discharge cycles $\left(n_{2}\right)$ of panel 2 must be carried out per, say, two consecutive cycles of panel 1 to achieve similar hysteresis windows for both panels. The calculus is performed by rounding the ratio between both currents to the closer integer number,

$$
n_{2}=\operatorname{round}\left(\frac{2 i_{\mathrm{PV} 2}}{i_{\mathrm{PV} 1}}\right)
$$

$n_{2} / 2$ sets the ratio between the periods of charge-discharge cycles for both PV panels. Fig. 5 shows the charge-discharge cycles for several values of $n_{2}$. Note that similar hysteresis windows are achieved for $v_{\mathrm{PV} 1}$ and $v_{\mathrm{PV} 2}$ in spite of their charge slopes being different. Other proportionality constants for the ratios of the charge-discharge periods (e.g. multiples of $1 / 3$, $1 / 4,1 / 5 \ldots$ ) could be selected. Lower values yield better time resolution to tune the proper switching period for each PV panel.

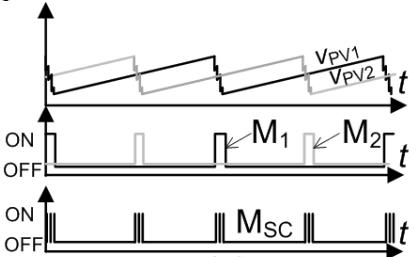

(a)

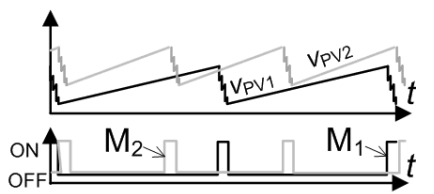

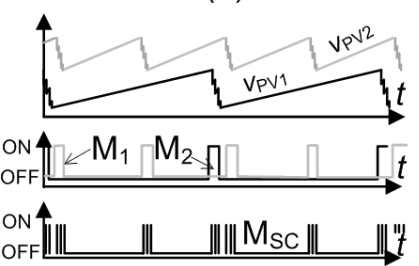

(c)

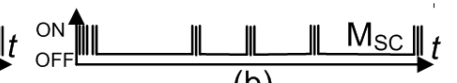

(b)
Fig. 5. Proposed TDM control for two PV panels to keep the hysteresis windows constant. (a) $n_{2}=2$, (b) $n_{2}=3$, (c) $n_{2}=4$ and $n_{2}=5$.

The switching converter cannot be directly controlled by a conventional hysteresis voltage comparator that indicates when the discharge state must start or finish in order to keep $v_{\mathrm{PV}}$ within the hysteresis cycle. Instead, a timer is also needed to synchronize the discharging states to avoid time overlapping. Fig. 6 shows a simplified control scheme that includes both control goals. A microcontroller (MCU) uses two output digital ports (POUT2 and POUT1) to control the switching converter and to select the input PV panel through an analog multiplexer. An embedded clock $\left(f_{\mathrm{CLK}}\right)$ drives the synchronization timer. On the other hand, an external comparator sets the low threshold value $\left(v_{\mathrm{TL}}\right)$ of $v_{\mathrm{PV}}$ during the discharge and indicates the MCU when the discharge must be stopped through PIN1 input digital port. An analog output (AN_OUT1) is used by the MCU to tune $v_{\mathrm{TL}}$.

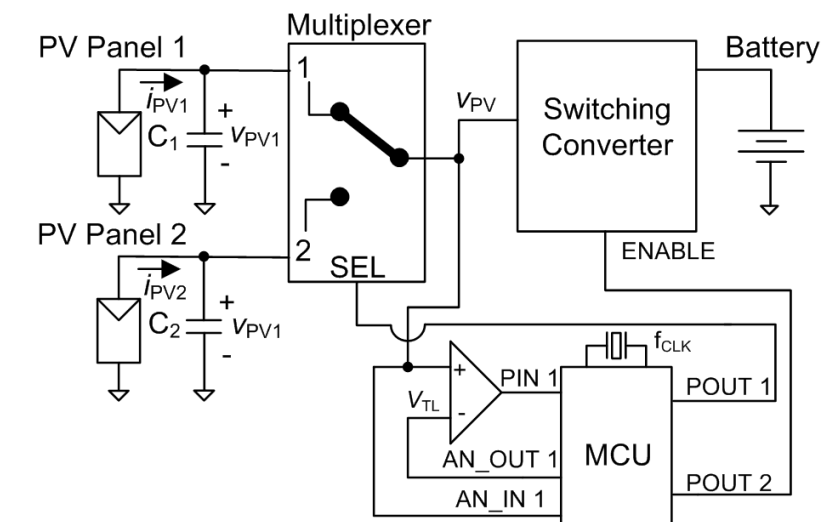

Fig.6. Simplified diagram of the proposed MIC photovoltaic system for two PV panels. 
Fig. 7 shows the time evolution of $v_{\mathrm{PV} 1}, v_{\mathrm{PV} 2}, v_{\mathrm{TL}}$, and the timer (Cycle) in two consecutive charge-discharge cycles of $v_{\mathrm{PV} 1}$. Note that $v_{\mathrm{TL}}$ is changed between $\mathrm{V}_{\mathrm{TL} 1}$ and $\mathrm{V}_{\mathrm{TL} 2}$ to set a proper threshold value for each panel. The timer resolution $\left(T_{\mathrm{CY}}\right)$ corresponds to the minimum time distance between the start times of two consecutive discharging states. To avoid time overlapping, $T_{\mathrm{CY}}$ must be longer than the time length needed by the switching converter to discharge the capacitor. Timer interrupts are used to set the start times of the discharging states and falling edge interrupts on PIN 1 indicate the end times. The timer is set to periodically count from 0 to $4 n_{2}-1$. The discharge states of PV panel 1 start when the cycle reaches 0 or $2 n_{2}$. On the other hand, the charge-discharge period of $v_{\mathrm{PV} 2}$ is $4 T_{\mathrm{CY}}$ and the first discharge is delayed by $T_{\mathrm{OFF} 2}$. To maximize the distance between two consecutive discharge states, $T_{\mathrm{OFF} 2}$ is selected to be $T_{\mathrm{CY}}$ for odd $n_{2}$ values and $2 T_{\mathrm{CY}}$ otherwise.

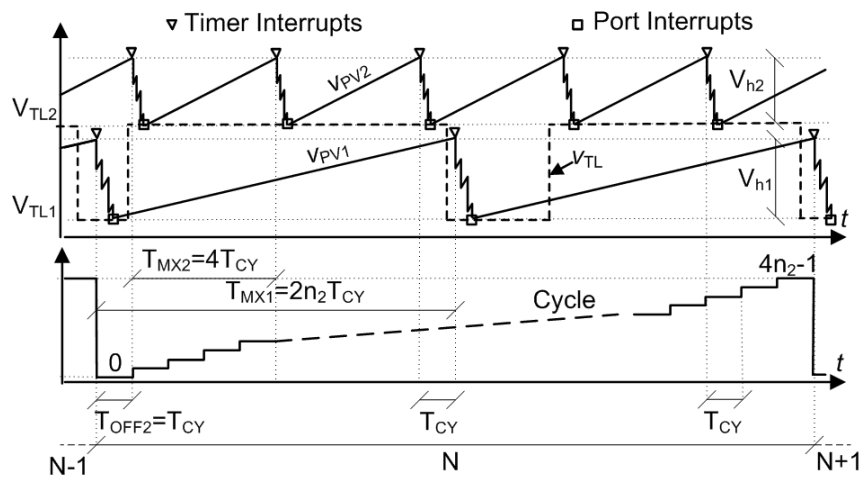

Fig. 7. Photovoltaic system timing diagram showing the time synchronization of discharging states for $\mathrm{n}_{2}=5$.

Four control variables $\left(n_{2}, V_{\mathrm{TL} 1}, V_{\mathrm{TL} 2}\right.$ and $\left.T_{\mathrm{CY}}\right)$ are tuned by the MCU to keep the hysteresis windows $\left(V_{\mathrm{h} 1}\right.$ and $\left.V_{\mathrm{h} 2}\right)$ close to $V_{\mathrm{h} \text { ref }}$ and, $v_{\mathrm{PV} 1}$ and $v_{\mathrm{PV} 1}$ around MPP $\left(V_{\mathrm{MPP} 1}\right.$ and $\left.V_{\mathrm{MPP} 2}\right)$. While $n_{2}$ is set to achieve similar hysteresis windows $\left(V_{\mathrm{h} 1} \approx V_{\mathrm{h} 2}\right), T_{\mathrm{CY}}$ sets that the average values match $V_{\mathrm{h} \text { ref }}$ and, $V_{\mathrm{TL} 1}$ and $V_{\mathrm{TL} 2}$ are selected as

$$
\begin{aligned}
& V_{\mathrm{TL} 1}=V_{\mathrm{MPP} 1}-\mathrm{V}_{\mathrm{h} \mathrm{ref}} / 2 \\
& V_{\mathrm{TL} 2}=V_{\mathrm{MPP} 2}-\mathrm{V}_{\mathrm{h} \mathrm{ref}} / 2
\end{aligned}
$$

$V_{\mathrm{MPP} 1}$ and $V_{\mathrm{MPP} 2}$ are periodically established by measuring the open-circuit voltage of PV panels with a fractional open circuit voltage (FOCV) control scheme [19][20]. An analog input (AN_IN1) of MCU measures the open-circuit voltage and also the high limit of the hysteresis windows to calculate $V_{\mathrm{h} 1}$ and $V_{\mathrm{h} 2}$. Measuring these voltage drops is simpler than using sensor currents to measure $i_{\mathrm{PV} 1}$ and $i_{\mathrm{PV} 2}$ and also, lets to sort PV panels and calculate $n_{2}$ in an equivalent way.

Control variables are updated every TDM control period, which corresponds to two consecutive charge-discharge cycles of $v_{\mathrm{PV} 1}$. The following relationships are established during $i=$ $1, \ldots, N, N+1, .$. control periods,

$$
\begin{gathered}
V_{\mathrm{h} 1}[i]=\frac{2 n_{2}[i]}{\mathrm{C}_{1}} i_{P V 1}[i] T_{C Y}[i] \\
V_{\mathrm{h} 2}[i]=\frac{4}{\mathrm{C}_{2}} i_{P V 2}[i] T_{C Y}[i]
\end{gathered}
$$

where, for the sake of simplicity, we assume $C_{1}=C_{2}$.
If we accept that $i_{\mathrm{PV} 1}[\mathrm{i}]$ and $i_{\mathrm{PV} 2}[\mathrm{i}]$ will hardly change for two consecutive control periods ( $N$ and $N+1)$, then

$$
V_{\mathrm{h} 1}[N+1]=V_{\mathrm{h} 1}[M] \frac{n_{2}[N+1]}{n_{2}[N]} \frac{V_{h 2}[N+1]}{V_{h 2}[N]}
$$

and from here it follows that to achieve $V_{\mathrm{h} 1}[N+1] \approx V_{\mathrm{h} 2}[N+$ 1] we need,

$$
n_{2}[N+1]=\operatorname{round}\left(\frac{\mathrm{V}_{\mathrm{h} 2}[N]}{\mathrm{V}_{\mathrm{h} 1}[N]} n_{2}[\mathrm{~N}]\right)
$$

On the other hand, $T_{\mathrm{CY}}[N+1]$ is tuned to fulfil

$$
V_{\mathrm{h} \mathrm{ref}}=\frac{V_{\mathrm{h} 1}[N+1]+V_{\mathrm{h} 2}[N+1]}{2}
$$

By replacing (4) and (5) in (8), it follows,

$$
T_{C Y}[N+1]=\frac{2 T_{\mathrm{CY}}[N] \mathrm{V}_{\mathrm{h} \mathrm{ref}}}{V_{h 1}[N] n_{2}[N+1] / n_{2}[N]+V_{h 2}[N]}
$$

\section{General time-multiplexing control.}

To extend the TDM control to $z_{\mathrm{PV}} \mathrm{PV}$ panels we need to find the right offset time delays $\left(T_{\mathrm{OFF} 2}, \ldots, T_{\mathrm{OFF} z_{P V}}\right)$ and discharge periods $\left(T_{\mathrm{MX} 1}, T_{\mathrm{MX} 2}, \ldots, T_{\mathrm{MX} z_{P V}}\right)$ that define the start times of the discharging states and prevent time overlapping.

Fig. 8 shows the time evolution of $v_{\mathrm{PVj}}$ for $j=1, . ., z_{\mathrm{PV}}$ and the definition of these time intervals. The same as in the previous section, the first design step is to sort PV panels from the lowest current to the highest current, which leads to

$$
i_{\mathrm{PV} Z_{P V}} \geq i_{\mathrm{PV} Z_{P V}-1} \geq \cdots \geq i_{\mathrm{PV} 1}
$$

and then to calculate the integer that sets the current ratios between PV panels,

$$
\begin{gathered}
n_{2}=\operatorname{round}\left(\frac{2 i_{\mathrm{PV} 2}}{i_{\mathrm{PV} 1}}\right) \\
n_{j}=\operatorname{round}\left(\frac{2^{j-1}}{n_{j-1} \ldots n_{2}} \frac{i_{\mathrm{PVj}}}{i_{\mathrm{PV} 1}}\right) \quad \text { for } j=3, \ldots, z_{\mathrm{PV}}
\end{gathered}
$$

where $n_{j}$ is the number of charge-discharge cycles performed by the $j$ PV panel per two cycles of the $j-1$ PV panel. Fig. 8 shows that four cycles are performed by the second panel $\left(n_{2}=4\right)$ per two cycles of the first panel. Similarly, three cycles of the third panel $\left(n_{3}=3\right)$ are performed per two cycles of second panel. Therefore, the following relation between the discharge periods of two consecutive panels results,

$$
T_{\mathrm{MX} \mathrm{j}}=\frac{n_{j+1}}{2} T_{M X \mathrm{j}+1} \quad \text { for } \quad j=1, \ldots \mathrm{ZPV}_{\mathrm{PV}}-1
$$

From (13), the discharge period of each PV panel is

$$
T_{\mathrm{MX} j}=\frac{n_{z_{\mathrm{PV}}} n_{z_{\mathrm{PV}}-1} \cdots n_{j+1}}{2^{z_{\mathrm{PV}}-j}} T_{M X z_{\mathrm{PV}}}
$$

For simplicity, we will consider a fixed number of $T_{\mathrm{CY}}$ cycles per each $T_{\mathrm{MX} z_{\mathrm{PV}}}$. This number does not depend on $n_{2}$, $n_{3}, \ldots, n_{Z_{P V}}$, and depends only on the number of PV panels $\left(T_{\mathrm{MX} Z_{P V}}=4 T_{C Y}\right.$, for $Z_{\mathrm{PV}}=2$ in section II.A). 


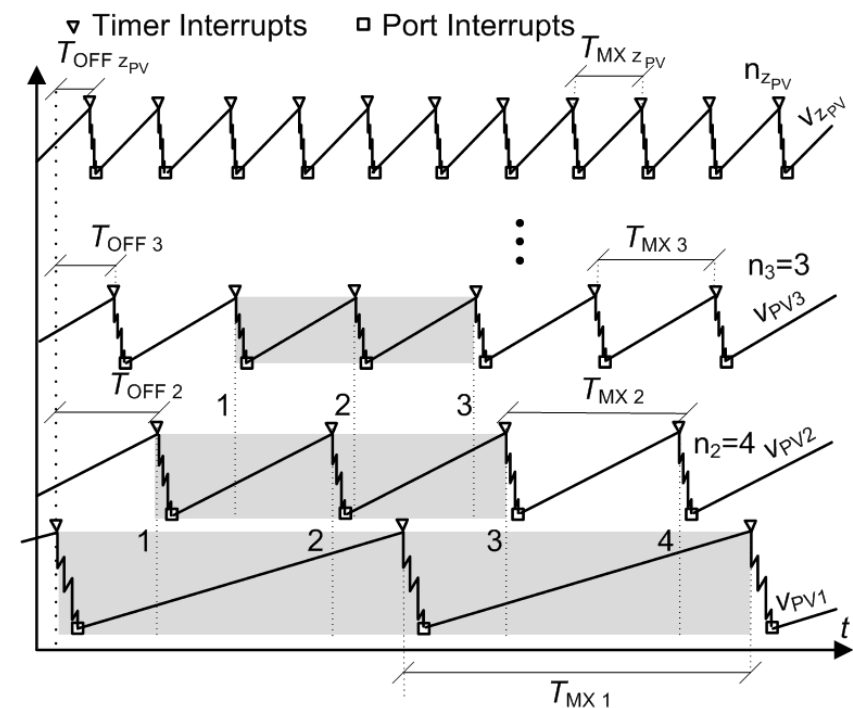

Fig. 8. PV system timing diagram that shows the time synchronization of discharge states for $n_{Z_{P V}}$ PV panels.

A design constraint to determine $T_{M X Z_{P V}}$ results from (14) by imposing that $T_{\mathrm{MXj}}$ must be a multiple of $T_{\mathrm{CY}}$ even when $j=1$ and $n_{2}, n_{3}, \ldots$ and $n_{Z_{P V}}$ are odd numbers. To fulfil this condition, $T_{\mathrm{MX} z_{p v}}$ must be proportional to $2^{Z_{P V}-1} T_{\mathrm{CY}}$. A second design constraint results by considering that the ratio between $T_{\mathrm{MX} j}$ and $T_{\mathrm{MX} Z_{P V}}$ could be an expression such as $n_{\text {odd }} / 2^{x}$, where $n_{\text {odd }}$ is an odd number and $x \in\left\{0, \ldots, z_{P V}-j\right\}$. Fig. 9 shows an example of this scenario wherein $T_{\mathrm{MXj}}=$ $5 / 2^{2} T_{\mathrm{MX} Z_{P V}}$. As a consequence, the discharge sequence of both PV panels is repeated every $2^{x} T_{\mathrm{MX} j}$. The start times of the $j$ panel discharging states are distributed inside a $T_{\mathrm{MX} Z_{P V}}$ period with a time interval $T_{\mathrm{MX} Z_{P V}} / 2^{x}$. In Fig. 9, four points are marked with " $x$ " that denote the equivalent position of the start times of $j$ panel discharging states during successive cycles of the $z_{\mathrm{PV}}$ panel. To avoid overlapping, it is assumed that these points $\left(T_{\mathrm{CY}}, 3 T_{\mathrm{CY}}, 5 T_{\mathrm{CY}}\right.$ and $\left.7 T_{\mathrm{CY}}\right)$ cannot be used to start the discharge of another PV panel and will set a minim threshold of $T_{\mathrm{CY}}$ cycles for $T_{\mathrm{MX} z_{P V}}$. By considering all PV panels in a worst-case scenario, it follows $T_{\mathrm{MX} Z_{P V}}>\left(2^{z_{P V}-1}+\right.$ $\left.\cdots+2^{1}+2^{0}\right) T_{\mathrm{CY}}=\left(2^{z_{P V}}-1\right) T_{\mathrm{CY}}$. Finally, from both design constraints the minimum $T_{\mathrm{MX} Z_{P V}}$ is

$$
T_{M X z_{p v}}=2^{Z_{P V}} T_{C Y}
$$

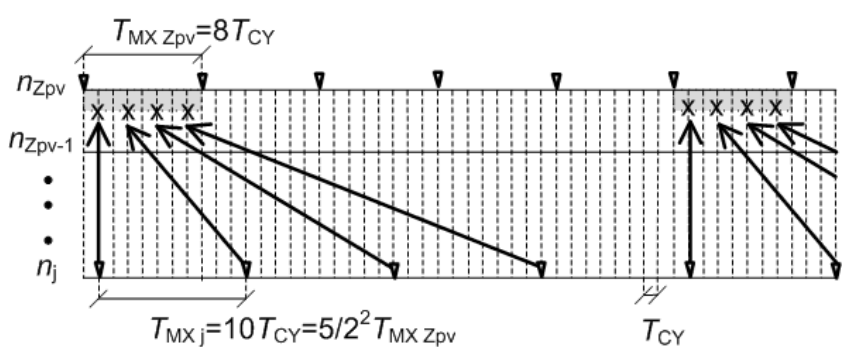

Fig. 9. Time distribution of start times of discharging states $(\boldsymbol{\nabla})$ for $\mathrm{Z}_{\mathrm{PV}}$ and $j$ $\mathrm{PV}$ panels, and their equivalent position (x) in a single $T_{M X Z_{P V}}$ period.

This value lets us to set the start times without overlapping if the following offset time delays are used,

$$
T_{\mathrm{OFF} j}=\left(2^{j-1}-1\right) T_{\mathrm{CY}} \quad \text { for } j=1, \ldots, \mathrm{ZPV}_{\mathrm{PV}}
$$

Proof: See Appendix A.

Fig. 10 shows the time distribution in the worst case scenario ( $n_{j}$ are odd numbers for $j=1, \ldots, z_{\mathrm{PV}}$ ) for five PV panels. Other $n_{j}$ values lead to different time distributions that are also included. An example is depicted in Fig. 10 where the equivalent start times of discharging states are marked with 0 for $n_{2}=3, n_{3}=5, n_{4}=4$ and $n_{5}=3$.

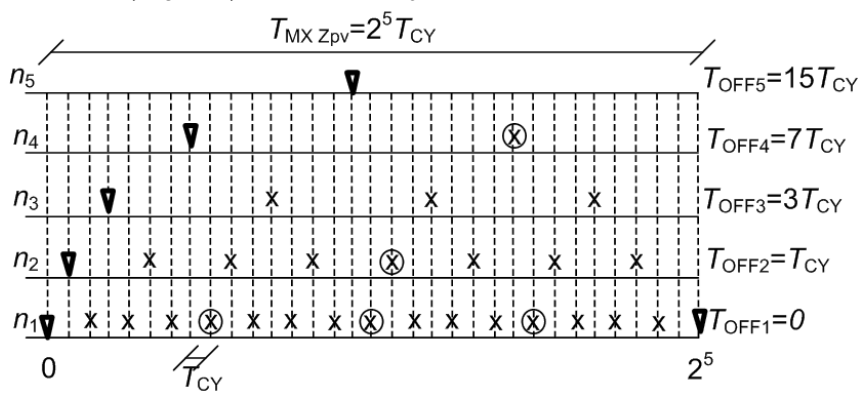

Fig. 10. Distribution of start times of discharging states ( $\mathbf{v})$ and their equivalent position (x) in a single $\mathrm{T}_{\mathrm{MX} \text { Zpv }}$ period for a worst-case scenario and five PV panels $\left(\mathrm{Z}_{\mathrm{PV}}=5\right)$. Equivalent start times $(O)$ for $n_{2}=3, n_{3}=5, n_{4}=4$ and $n_{5}=3$.

The control variables $\left(n_{2}, n_{3}, \ldots, n_{z_{P V}}, V_{T L 1}, \ldots, V_{T L z_{P V}}\right.$ and $\left.T_{\mathrm{CY}}\right)$ are tuned by the MCU using the same control goals as in Section II.A. The following relations between the control variable of two consecutive control periods are obtained for $j=$ $2, \ldots, z_{P V}$,

$$
\begin{aligned}
& n_{j}[N+1]=\operatorname{round}\left(\frac{V_{\mathrm{h} j}[N]}{V_{\mathrm{h} 1}[N]} n_{j}[N] \prod_{x=2}^{\mathrm{j}-1} \frac{n_{x}[N]}{n_{X}[N+1]}\right) \\
& V_{\mathrm{TL} j}=V_{\mathrm{MPP} j}-V_{\mathrm{h} \text { ref }} / 2 \\
& T_{\mathrm{CY}}[N+1]=\frac{\mathrm{z}_{\mathrm{PV}} \mathrm{V}_{\mathrm{h} \mathrm{ref}} T_{\mathrm{CY}}[N]}{\sum_{j=1}^{\mathrm{zPV}}\left(V_{\mathrm{hj}}[N] \prod_{\mathrm{x}=\mathrm{j}+1}^{\mathrm{PVV}} n_{\mathrm{x}}[N+1] / n_{\mathrm{x}}[N]\right)}
\end{aligned}
$$

These values are updated every $2^{\mathrm{Z}_{\mathrm{PV}}-1} \mathrm{~T}_{\mathrm{MX} 1}$ cycles. Note that the switching pattern is repeated after this time length.

\section{DESIGN CONSTRAINT}

The aim of TDM control is that several power sources share a single switching converter, which implies that the power processing capability of the converter must exceed the sum of those power sources. Further, to avoid time overlapping, the switching converter cannot be active fulltime hence extra power capability is needed. To calculate the required power capability we will assume, for the sake of simplicity, that all PV panels have the same performance, $C_{j}=C, I_{\mathrm{DSCH}} \gg i_{\mathrm{PVj}}$ and $T_{\mathrm{MX}} \gg T_{\mathrm{CY}}$ for $j=1, \ldots, z_{\mathrm{PV}}$. The minimal power required is calculated from the minimum $I_{\mathrm{DSCH}}$ that is needed to discharge each input capacitor on time.

$$
T_{\mathrm{CY}}>\frac{C_{j} V_{\mathrm{hj}}}{I_{\mathrm{DSCH}}-i_{\mathrm{PVj}}} \approx \frac{C V_{\mathrm{h} \mathrm{ref}}}{I_{\mathrm{DSCH}}}
$$


and the relation that sets the control loop that fixes the required and remain in sleep mode otherwise.

hysteresis window to $V_{\mathrm{h} \text { ref }}$ is

$$
\begin{aligned}
& V_{\mathrm{h} \mathrm{ref}}=\frac{\sum_{j=1}^{z_{P V}} V_{\mathrm{hj}}}{Z_{\mathrm{PV}}}=\frac{\sum_{j=1}^{z_{P V}} \frac{i_{\mathrm{PVj}}\left(T_{\mathrm{MX}}-T_{\mathrm{CY}}\right)}{C_{\mathrm{j}}}}{Z_{\mathrm{PV}}} \\
& \approx \frac{\sum_{j=1}^{Z_{P V}} i_{\mathrm{PVj}} 2^{j} n_{\mathrm{zPV}} n_{\mathrm{zPV}-1} \ldots n_{\mathrm{j}+1}}{Z_{\mathrm{PV}} C} T_{\mathrm{CY}}
\end{aligned}
$$

By substituting the expression of $T_{\mathrm{CY}}$ obtained from (23) in (22), we obtain

$$
I_{\mathrm{DSCH}}>\frac{\sum_{j=1}^{z_{P V}} i_{\mathrm{PVj}} 2^{j} n_{\mathrm{z}_{\mathrm{PV}}} n_{\mathrm{z}_{\mathrm{PV}}-1} \ldots n_{\mathrm{j}+1}}{z_{\mathrm{PV}}} \approx 2^{z_{P V}} i_{\mathrm{PV} z_{P V}}
$$

where the approximation results by considering the rounding relations in (11) and (12) exact.

The efficiency $(\eta)$ of the proposed time multiplexing control, defined as the ratio between the sum of $i_{\mathrm{PVj}}$ and $I_{\mathrm{DSCH}}$, is bounded by (24) and can be expressed as

$$
\eta \equiv \frac{\sum_{j=1}^{z_{P V}} i_{P V j}}{I_{D S C H}}<\frac{Z_{P V} i_{\mathrm{PV}_{P V}}}{I_{D S C H}}<\frac{z_{P V}}{2^{z_{P V}}}
$$

This limit must be taken into account when selecting the switching converter during the design process. Note that $z_{P V} / 2^{z_{P V}}$ decreases for an increasing number of multiplexed PV panels. A large $z_{\mathrm{PV}}$ could make the proposed multiplexed technique unfeasible if the resulting efficiency is so small that implies a huge switching converter. This design constraint could be relaxed if the possible values of $n_{2}, n_{3}, \ldots, n_{z_{P V}}$ are restricted. The use of even numbers would let to reduce discharge periods but would worsen the time resolution to tune $T_{\mathrm{MX} 2}, \ldots$ and $T_{\mathrm{MX} z_{P V}}$.

\section{PROOF OF CONCEPT}

In order to demonstrate the feasibility of the proposed TDM control, we have implemented a prototype of low-power MPPT with two non-aligned PV panels (SLMD121H04) (Fig. 11). The solar energy harvester was part of a sensor node which MCU (MSP430FG4618) was also used to control the multiplexer (FDF1320) and the switching converter (MAX1795) to select the input capacitor and starting times for discharge. For these components, $I_{\mathrm{DSCH}}(250 \mathrm{~mA})$ and $i_{\mathrm{PV}}$ (50 mA, STC) fulfil the design condition (25). MAX1795 would not work if more SLMD121H04 panels were used. In that case, MAX1797 $\left(I_{\mathrm{DSCH}}=1 \mathrm{~A}\right)$ could manage up to four SLMD121H04 panels.

The voltage comparator has been implemented with a lowpower op amp (EL8176) and several resistors (10 M $\Omega$ and $390 \mathrm{k} \Omega$ ) that fit the switching thresholds in the voltage range of $V_{\text {MPP. }}$. The digital input port (P2.1) detects the falling edge and issues interrupts that stop the discharge states. Timer interrupts are issued by an embedded timer that is driven by a $32 \mathrm{kHz}$ clock (ACLK). This low-power clock and the low-power modes of MCU (LPM3) result in a very low-power consumption of the overall system that is essential in this kind of applications [12]. MCU and EL8176 are active only when

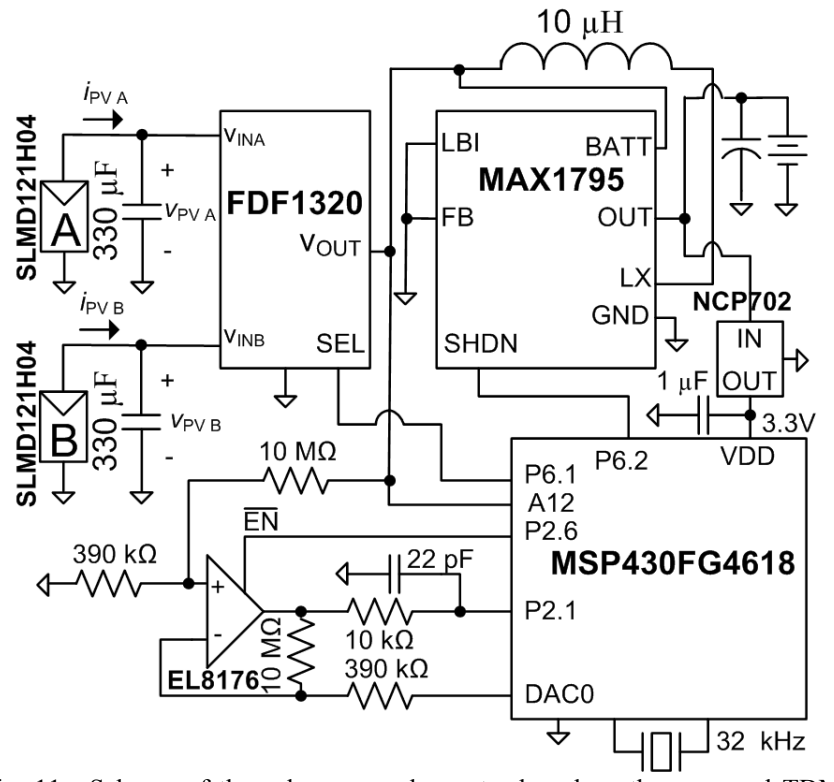

Fig. 11. Scheme of the solar energy harvester based on the proposed TDM scheme for two PV panels.

The prototype has been tested in the laboratory under controlled irradiance on each PV panel. Figs. 12-15 show the bias voltage of the input capacitors $\left(v_{\mathrm{PV}} \mathrm{A}\right.$ and $v_{\mathrm{PV}}$ B $)$ and the control signals of the switching converter (SHDN) and multiplexer (SEL) for different operating conditions. Specifically, Fig. 12 shows signal waveforms when the irradiance on both PV panels is similar and $n_{2}=2$. The input capacitors of both PV panels are discharged at the same frequency by keeping $V_{\mathrm{h} 1}$ and $V_{\mathrm{h} 2}$ around $V_{\mathrm{h} \text { ref }}(200 \mathrm{mV})$. SEL is hold at high or low state depending on whether PV panel A or B must be respectively discharged. SHDN is driven to low state to activate the switching converter and discharge the input capacitor.

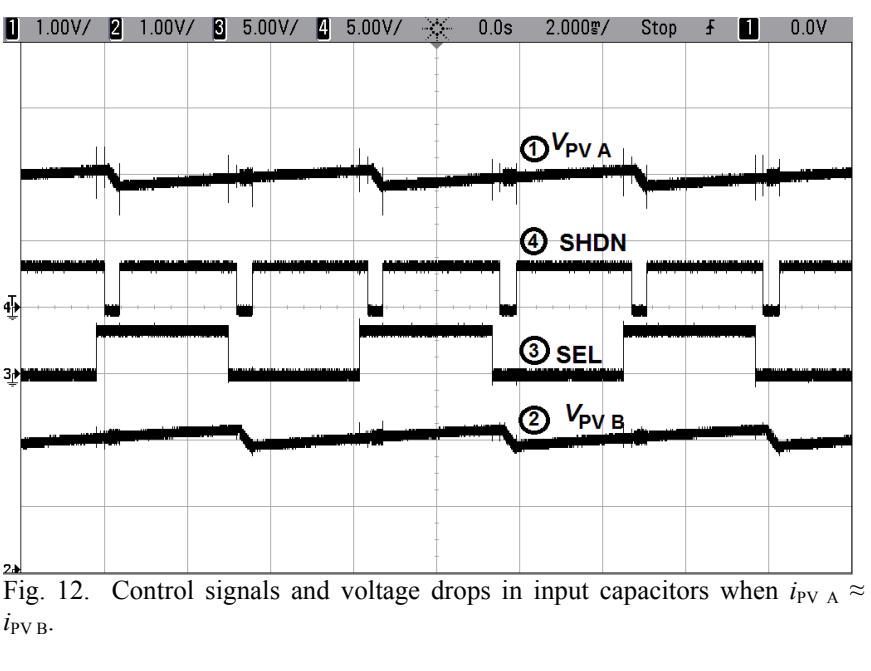

Fig. 13 shows the same signals when the irradiance on panel $B$ has been increased by about $5 / 2$ times. The controller tunes $T_{\mathrm{CY}}$ and $n_{\mathrm{B}}\left(=n_{2}\right)$ to keep $V_{\mathrm{h} 1}$ and $V_{\mathrm{h} 2}$ around $V_{\mathrm{h} \text { ref. In this }}$ case, five charge-discharge cycles of panel $\mathrm{B}$ are performed per two cycles of panel A $\left(n_{2}=5\right)$. 


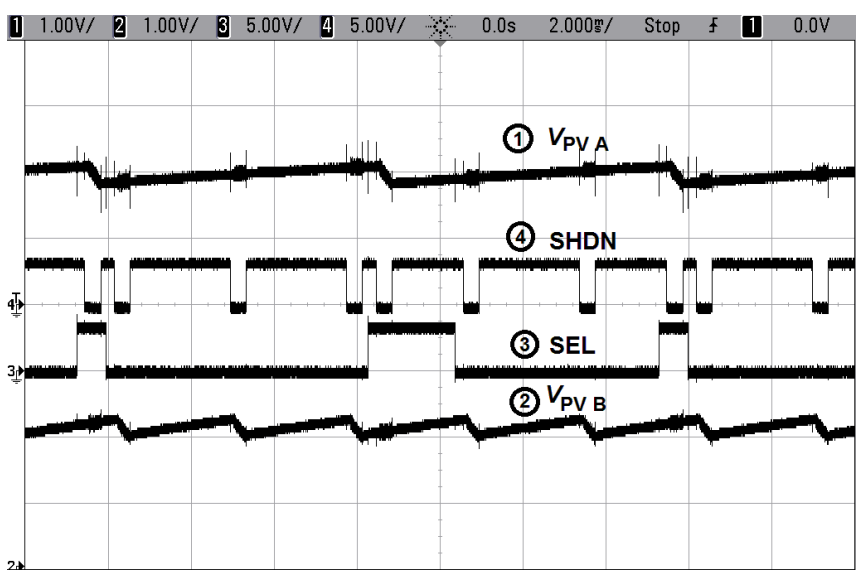

Fig. 13. Control signals and voltage drops in input capacitors when $5 i_{\mathrm{PV} \mathrm{A}} \approx i_{\mathrm{PV}}$

The resulting waveforms when the irradiance on panel $\mathrm{A}$ is increased by $6 / 2$ times are shown in Fig. 14. Now the controller detects that $i_{\mathrm{PV}}$ is higher than $i_{\mathrm{PV}}$, and panels $\mathrm{A}$ and $\mathrm{B}$ are identified as panel 2 and $1\left(n_{2}=n_{\mathrm{A}}=6\right)$ respectively, and panel A performs six charge-discharge cycles per two cycles of panel B.

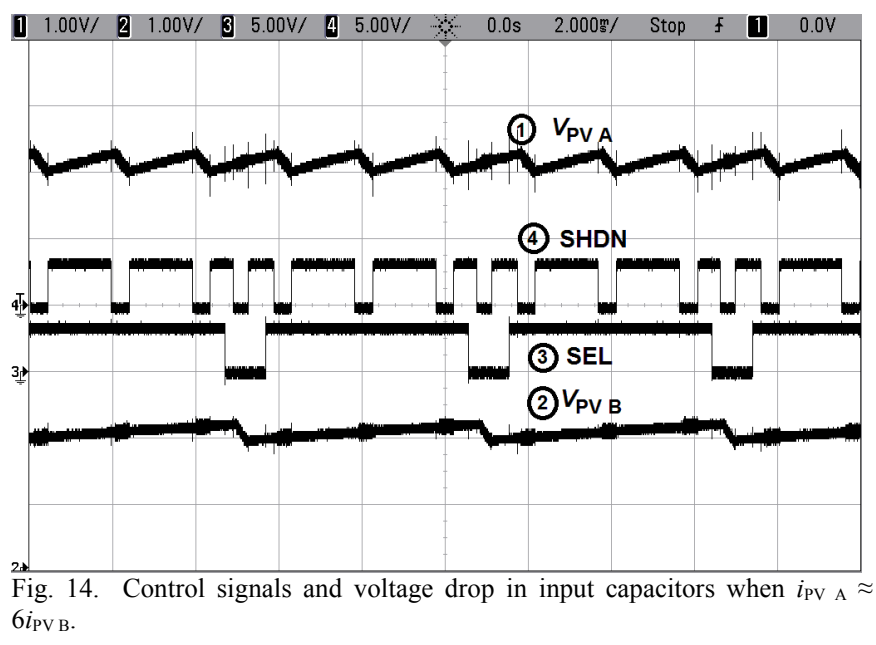

The values of $V_{\mathrm{TL} 1}$ and $V_{\mathrm{TL} 2}$ are periodically updated according to the variation of $V_{\mathrm{MPP} 1}$ and $V_{\mathrm{MPP} 2}$ caused by the changing incident irradiance. The FOCV control method is used to calculate $V_{\mathrm{MPP} 1}$ and $V_{\mathrm{MPP} 2}$ from the measured open circuit voltage $\left(V_{\text {oc }}\right)$ of each PV panel $\left(V_{\mathrm{MPP}}=0.8 V_{\text {oc }}\right)$. Fig. 15 shows the sampling time of these voltages. After a fixed number of charge-discharge cycles of panel $\mathrm{A}$, no discharging states are allowed for five consecutive cycles to let PV panels reach the open circuit voltages $V_{\mathrm{ocA}}$ and $V_{\mathrm{ocB}}$, which are captured by analog input A12.

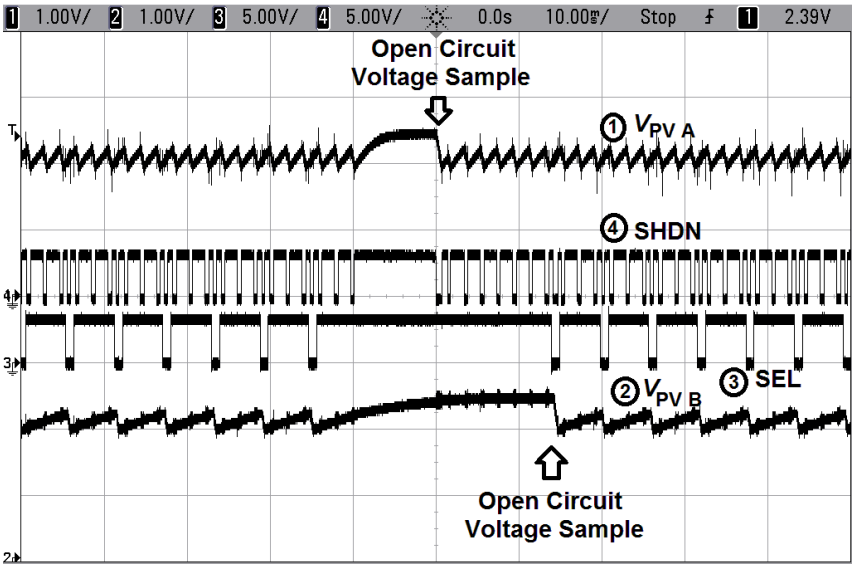

Fig. 15. Control signal and voltage drops in input capacitors when open circuit voltages are sampled to calculate $\mathrm{V}_{\mathrm{MPP}}$.

\section{CONCLUSIONS}

A new TDM control has been proposed that allows several non-aligned PV panels to share a single MPPT. In contrast to previous TDM control schemes, the proposed control algorithm allows us to separately tune the periods of charge-discharge cycles of each PV panel and to implement PFM, which is required in low-power converters. This way, a single switching converter can bias each PV panel around its MPP with a fixed hysteresis window. A synchronization timer, whose resolution is delimited by the maximum discharge states length, sets a periodic control pattern that avoids time overlapping. This is achieved by keeping the ratio between the periods of chargedischarge cycles to multiples of $1 / 2$. Similar control algorithms result from other proportionality constants, which can be used to improve the resolution of periods' tuning.

A design constraint analysis has concluded that the current discharge capability of switching converter must be higher than the sum of the currents generated by PV panels multiplied by $2^{Z_{P V}} / z_{P V}$, where $z_{P V}$ is the number of panels. That makes the described TDM scheme unfeasible for a large number of panels since a huge switching converter would be required. In that case, we suggest restricting the ratio between the chargedischarge cycles to even numbers and reducing periods' length.

The proposed TDM algorithm has been conceived for cost reduction in low-power autonomous sensor nodes. As a proof of concept, a MPPT has been implemented for two PV panels. No current sensor is used and a single MCU controls the energy harvester and performs the other tasks of the sensor node. It can be readily applied to low-power moveable nodes, such as sensor buoy systems in river or lakes, where nonaligned PV panels are required. In the case of static nodes, the use of non-aligned PV panels lets to reduce the daily discharge depth of energy storage devices hence extending the lifespan of batteries or allowing their replacement by supercapacitors. 


\section{APPENDIX A}

\section{Proof Of Not Overlapping Discharge States}

The start time of discharging state of two PV panels $\left(t_{i}\right.$ and [8] $t_{j}$ ) are given by

$$
\begin{aligned}
& t_{i}=T_{O F F i}+z_{i} T_{M X i} \\
& t_{j}=T_{O F F j}+z_{j} T_{M X j}
\end{aligned}
$$

where $z_{\mathrm{i}}$ and $z_{\mathrm{j}}$ are integer numbers, $i$ is considered higher [10] than $j$, and $T_{\mathrm{OFF}}$ and $T_{\mathrm{MX}}$ are given by (14)-(16).

Equating (26) and (27) results that if $t_{\mathrm{i}}$ and $t_{\mathrm{j}}$ ever matches then two integer numbers $\left(z_{\mathrm{i}}\right.$ and $\left.z_{\mathrm{j}}\right)$ exist that satisfies

$$
2^{i-j}=\frac{1+2 z_{j} \prod_{x=j+1}^{z_{P V}} n_{x}}{1+2 z_{i} \prod_{x=i+1}^{z_{P V}} n_{x}}
$$

This relationship can never be satisfied because the left side term is an even number whereas the right side term is quotient of two odd numbers.

\section{REFERENCES}

[1] C. Albaladejo, F. Soto, R. Torres, P. Sanchez, and J. A. Lopez, "A lowcost sensor buoy system for monitoring shallow marine environments," Sensors, vol. 12, no. 7, pp. 9613-9634, Jul. 2012.

[2] R, Bondade, Y. Zhang, B. Wei, T. Gu, H. Chen, and D. Brian Ma, "Integrated Auto-Reconfigurable Power-Supply Network with Multidirectional Energy Transfer for Self-Reliant Energy-Harvesting Applications," IEEE Trans. Ind. Electron., vol. 63, no.5, pp.2850-2861, May 2016.

[3] T. Guena, and P. Leblanc, "How Depth of Discharge Affects the Cycle life of Lithium-Metal-Polymer Batteries," Telecom. Energy Conf., 2006, INTELEC'06, Sept., 2006.

[4] D. Linden and T. B. Reddy, "Handbook of batteries," $2^{\text {nd }}$ edition, New York 2010 .

[5] C. Zhu, L. T. Yang, L. Shu, V. C. M. Leung, T. Hara, and S. Nishio, "Insights of top-k query in duty-cycled wireless sensor networks," IEEE Transactions on Industrial Electronics, vol. 62, no. 2, pp. 1317-1328, Feb. 2015.

[6] W. Huang and J. A. A. Qahouq, "Energy Sharing Control Scheme for State-of-Charge Balancing of Distributed Battery Energy Storage System," IEEE Trans. Ind. Electron., vol. 62, no. 5, pp. 2764-2776, May. 2015.
[7] J. A. A. Qahouq and Y. Jiang, "Distributed photovoltaic solar system architecture with single-power inductor single-power converter and single-sensor single maximum power point tracking controller," IET Power Electron., vol. 7, no. 10, pp. 2600-2609, March 2014.

W. Cai and F. Yi, "Topolgy Simplification Method based on Switch Multiplexing Technique to Deliver DC-DC-AC Converters for microgrid Applications", Energy Conversion Congress and Exposition (ECCE), pp. 6667-6674, 2015.

[9] L. Solero, A. Lidozzi and J. A. Pomilio, "Design of Multiple-Input Power Converter for Hybrid Vehicles," IEEE Trans. Power Electron., vol. 20, no. 5, pp. 1107-1016, Sept. 2005.

10] J. A. A. Qahouq and Y. Jiang, "Distributed photovoltaic solar system architecture with single-power inductor single-power converter and single-sensor single maximum power point tracking controller," IET Power Electron., vol. 7, Iss. 10, pp. 2600-209, 2014.

[11] L. Xian and Y. Wang,. "Exact steady-state analysis in multiple-input converters applied with diverse time-sharing switching schemes", A neural network-based low-cost solar irradiance sensor," IET Power Electron., vol. 8, Iss. 5, pp. 724-734, 2015

[12] H. Behjati and A. Davoudi, "A multiple-input multiple-output DC-DC converter," IEEE Trans. Ind. Appl., 2013, 49, pp. 1464-1479.

[13] R. Ahmadi, H. Zargarzadeh, and M. Ferdowsi "Nonlinear power sharing controller for a double-input H-bridge-based buck-boost - buck-boost converter," IEEE Trans. Power Electron., 2013, 28, pp. 2402-2414.

[14] Y. Dongsheng, Y. Min and R. Xinbo, "One-cycle control for a doubleinput DC/DC converter," IEEE Trans. Power Electron., 2012, 27, pp. 4646-4655.

[15] B. Sahu and G. A. Rincon-Mora, "An Accurate, Low-Voltage, CMOS Switching Power Supply With Adaptive On-Time Pulse-Frequency Modulation (PFM) Control," IEEE Trans. on Circuits and Systems. vol. 54, pp. 312-321, Feb. 2007.

16] O. Lopez-Lapena, M. T. Penella and M. Gasulla, "A New MPPT Method for Low-Power Solar Energy Harvesting," IEEE Trans. Ind. Electron., vol.57, no.9, pp.3129-3138, Sept. 2010.

17] O. Lopez-Lapeña, M. T. Penella, and M. Gasulla, "A closed-loop maximum power point tracker for subwatt photovoltaic panels," IEEE Trans. Ind. Electron., vol. 59, no. 3, pp. 1588-1596, Mar. 2012.

[18] K. Zakir Ahmed, and S. Mukhopadhyay, "A Wide Conversion Ratio, Extended Input 3.5- $\mu$ A Boost Regulator With 82\% Efficiency for LowVoltage Energy Harvesting." IEEE Trans. Power Electron., vol.29, no.9, pp.4776-4786,Sept. 2014

[19] O. Lopez-Lapena and M. T. Penella, "Low-power FOCV MPPT controller with automatic adjustment of the sample \& hold," Electron. Lett., vol. 48, no. 20, pp. 1301-1303, 2012.

[20] W. Y. Toh, Y. K. Tan, W. S. Koh and L. Siek, "Autonomous Wearable Sensor Nodes With Flexible Energy Harvesting,” IEEE Sensors Journal, vol. 14, no. 7, pp. 2299-2306, July 2014. 